KYUNGPOOK Math. J. 55(2015), 355-372

http://dx.doi.org/10.5666/KMJ.2015.55.2.355

pISSN 1225-6951 eISSN 0454-8124

(c) Kyungpook Mathematical Journal

\title{
On the Fibonacci Almost Convergent Sequence Space and Fi- bonacci Core
}

\author{
Serkan Demiriz* \\ Department of Mathematics, Gaziosmanpasa University, Tokat, 60240, Turkey \\ e-mail : serkandemiriz@gmail.com \\ EMraH EvREN KARA \\ Department of Mathematics, Duzce University, Duzce, Turkey \\ e-mail : eevrenkara@hotmail.com \\ METIN BAŞARIR \\ Department of Mathematics, Sakarya University, Sakarya, Turkey \\ e-mail : basarir@sakarya.edu.tr
}

ABSTRACT. In the present paper, by using the Fibonacci difference matrix, we introduce the almost convergent sequence space $\widehat{c}^{f}$. Also, we show that the spaces $\widehat{c}^{f}$ and $\widehat{c}$ are linearly isomorphic. Further, we determine the $\beta$-dual of the space $\widehat{c}^{f}$ and characterize some matrix classses on this space. Finally, Fibonacci core of a complex-valued sequence has been introduced, and we prove some inclusion theorems related to this new type of core.

\section{Introduction}

Let $\omega$ be the set of all complex sequences $x=\left(x_{k}\right)_{k=0}^{\infty}$ and $c_{0}, c$ and $\ell_{\infty}$ be the sets of all null, convergent and bounded sequences, respectively. Also by $b s$ and $c s$, we denote the spaces of all bounded and convergent series, respectively. Throughout this paper all infinite sequences and matrices are assumed to be indexed by $\mathbb{N}=\{0,1,2, \ldots\}$. For example, $\left(x_{k}\right)_{k \in \mathbb{N}}$ will be denoted simply as $\left(x_{k}\right)$. Also, for simplicity in notation, the summation without limits runs from 0 to $\infty$.

Let $X, Y$ be any two sequence spaces and $A=\left(a_{n k}\right)$ be an infinite matrix of real numbers $a_{n k}$, where $n, k \in \mathbb{N}$. Then, we say that $A$ defines a matrix mapping

\footnotetext{
* Corresponding Author.

Received December 22, 2013; accepted May 1, 2014.

2010 Mathematics Subject Classification: 46A45, 40A05, 46A35.

Key words and phrases: Sequence spaces, almost convergence, Fibonacci matrix, $\beta$-dual, matrix transformations, core theorems.
} 
from $X$ into $Y$, and we denote it by writing $A: X \rightarrow Y$, if for every sequence $x=\left(x_{k}\right) \in X$ the sequence $A x=\left((A x)_{n}\right)$, the $A$-transform of $x$, is in $Y$, where

$$
(A x)_{n}=\sum_{k} a_{n k} x_{k}, \quad(n \in \mathbb{N}) .
$$

By $(X: Y)$, we denote the class of all matrices $A$ such that $A: X \rightarrow Y$. Thus, $A \in(X: Y)$ if and only if the series on the right-hand side of (1.1) converges for each $n \in \mathbb{N}$ and every $x \in X$, and we have $A x=\left\{(A x)_{n}\right\}_{n \in \mathbb{N}} \in Y$ for all $x \in X$. A sequence $x$ is said to be $A$ - summable to $\alpha$ if $A x$ converges to $\alpha$ which is called as the $A$-limit of $x$. If $X$ and $Y$ are equipped with the limits $X-\lim$ and $Y-\lim$, respectively, $A \in(X: Y)$ and $Y-\lim _{n} A_{n}(x)=X-\lim _{k} x_{k}$ for all $x \in X$, then we say that $A$ regularly maps $X$ into $Y$ and write $A \in(X: Y)_{\text {reg }}$.

A matrix $A=\left(a_{n k}\right)$ is called a triangle if $a_{n k}=0$ for $k>n$ and $a_{n n} \neq 0$ for all $n \in \mathbb{N}$. It is trivial that $A(B x)=(A B) x$ holds for the triangle matrices $A, B$ and a sequence $x$. Further, a triangle $U$ uniquely has an inverse $U^{-1}=V$ that is also a triangle matrix. Then, $x=U(V x)=V(U x)$ holds for all $x \in \omega$.

A linear functional $B$ on $\ell_{\infty}$ is called a Banach limit if

(1) $B \geq 0$, i.e. $B(x) \geq 0$ for $x \geq 0$ and $B(e)=e$, where $e=(1,1,1, \ldots)$.

(2) $B(T x)=B(x)$ for all $x \in \ell_{\infty}$, where $T$ is the shift operator, that is, $T\left(x_{0}, x_{1}, \ldots\right)=\left(x_{1}, x_{2}, \ldots\right)$.

The existence of Banach limits was proven by Banach [1] in his book. It follows from the definition, that $B(x)=\lim _{n \rightarrow \infty} x_{n}$ for every $x=\left(x_{n}\right) \in c$. A sequence $x \in \ell_{\infty}$ is called almost convergent to the generalized limit $\alpha$ if all Banach limits of $x$ are $\alpha$ [2], and denoted by $\widehat{c}-\lim x_{k}=\alpha$. Let $\widehat{c}$ denote the set of all almost convergent sequences. Lorentz [2] proved that

$$
\widehat{c}=\left\{x=x_{k} \in \omega: \exists \alpha \in \mathbb{C} \ni \lim _{m \rightarrow \infty} \sum_{j=0}^{m} \frac{x_{n+k}}{m+1}=\alpha \text { uniformly in } n\right\} .
$$

It is known that $\widehat{c}$ is a Banach space with the norm [3]

$$
\|x\|_{\widehat{c}}=\sup _{m, n \in \mathbb{N}}\left|\sum_{j=0}^{m} \frac{x_{n+k}}{m+1}\right| .
$$

The domain $\widehat{c}_{A}$ of an infinite matrix $A$ in the almost convergent sequence space $\widehat{c}$ is defined by

$$
\widehat{c}_{A}=\left\{x=x_{k} \in \omega: A x \in \widehat{c}\right\} .
$$

In the literature, by using the some special triangular matrices, new almost convergent sequence spaces have been defined by several authors. For example, the spaces $\widehat{c}_{R^{t}}, \widehat{c}_{C}, \widehat{c}_{B(r, s)}, \widehat{c}_{B(r, s, t)}$ have been studied in $[4,5,6,7]$, respectively, where $R^{t}$ is the Riesz mean, $C$ is the Cesàro matrix of order one, and $B(r, s)=\left\{b_{n k}(r, s)\right\}$ and 
$B(r, s, t)=\left\{b_{n k}(r, s, t)\right\}$ are the generalized difference matrices respectively defined by

$$
b_{n k}(r, s)=\left\{\begin{array}{lc}
r, & k=n \\
s, & k=n-1 \\
0, & \text { otherwise }
\end{array} \text { and } b_{n k}(r, s, t)=\left\{\begin{array}{lc}
r, & k=n \\
s, & k=n-1 \\
t, & k=n-2 \\
0, & \text { otherwise }
\end{array}\right.\right.
$$

for all $k, n \in \mathbb{N}$. Recently, Kara and Elmaağaç [8], by using the $u$-difference matrix, defined $u$-difference almost sequence space $\hat{c}^{u}$, where $u$-difference matrix is the matrix $A^{u}=\left(a_{n k}^{u}\right)$ is defined by

$$
a_{n k}^{u}=\left\{\begin{array}{cc}
(-1)^{n-k} u_{k}, & n-1 \leq k \leq n \\
0, & 0 \leq k<n-1 \text { or } k>n
\end{array}\right.
$$

for all $k, n \in \mathbb{N}$. Also, we refer the reader to $[9,10,11,12,13,14,15,16,17,18$, $19,20,21]$ for a wide perspective on the concept of almost convergence and some generalizations and relations with matrix methods.

Let $x=\left(x_{k}\right)$ be a sequence in $\mathbb{C}$, the set of all complex numbers, and $R_{k}$ be the least convex closed region of complex plane containing $x_{k}, x_{k+1}, x_{k+2}, \ldots$. The Knopp Core (or $\mathcal{K}$ - core) of $x$ is defined by the intersection of all $R_{k}(k=1,2, \ldots)$, (see [22], pp.137). In [23], it is shown that

$$
\mathcal{K}-\operatorname{core}(x)=\bigcap_{z \in \mathbb{C}} B_{x}(z)
$$

for any bounded sequence $x$, where $B_{x}(z)=\left\{w \in \mathbb{C}:|w-z| \leq \lim \sup _{k}\left|x_{k}-z\right|\right\}$.

Let $E$ be a subset of $\mathbb{N}$. The natural density $\delta$ of $E$ is defined by

$$
\delta(E)=\lim _{n} \frac{1}{n}|\{k \leq n: k \in E\}|
$$

where $|\{k \leq n: k \in E\}|$ denotes the number of elements of $E$ not exceeding $n$. A sequence $x=\left(x_{k}\right)$ is said to be statistically convergent to a number $l$, if $\delta\left(\left\{k:\left|x_{k}-l\right| \geq \varepsilon\right\}\right)=0$ for every $\varepsilon$. In this case we write $s t-\lim x=l,[24]$. By st we denote the space of all statistically convergent sequences.

In [25], the notion of the statistical core (or st-core) of a complex valued sequence has been introduced by Fridy and Orhan and it is shown for a statistically bounded sequence $x$ that

$$
s t-\operatorname{core}(x)=\bigcap_{z \in \mathbb{C}} C_{x}(z),
$$

where $C_{x}(z)=\left\{w \in \mathbb{C}:|w-z| \leq s t-\lim \sup _{k}\left|x_{k}-z\right|\right\}$. The core theorems have been studied by many authors. For instance see $[26,27,28,29,30]$ and the others.

The sequence $\left(f_{n}\right)$ of Fibonacci numbers defined by the linear recurrence equalities

$$
f_{0}=f_{1}=1 \text { and } f_{n}=f_{n-1}+f_{n-2} \text { with } n \geq 2 .
$$


Fibonacci numbers have many interesting properties and applications in arts, sciences and architecture. For example, the ratio sequences of Fibonacci numbers converges to the golden ratio which is important in sciences and arts. Also, some basic properties of sequences of Fibonacci numbers [31] are given as follows:

$$
\begin{aligned}
& \lim _{n \rightarrow \infty} \frac{f_{n+1}}{f_{n}}=\frac{1+\sqrt{5}}{2}=\varphi \text { (Golden Ratio), } \\
& \sum_{k=0}^{n} f_{k}=f_{n+2}-1 \text { for each } n \in \mathbb{N}, \\
& \sum_{k} \frac{1}{f_{k}} \text { converges, } \\
& f_{n-1} f_{n+1}-f_{n}^{2}=(-1)^{n+1} \text { for all } n \geq 1
\end{aligned}
$$

Recently, Kara [32] has defined the sequence space $\ell_{p}(\widehat{F})$ as follows:

$$
\ell_{p}(\widehat{F})=\left\{x \in \omega: \widehat{F} x \in \ell_{p}\right\}, \quad(1 \leq p \leq \infty),
$$

where $\widehat{F}=\left(\widehat{f}_{n k}\right)$ is the double band matrix defined by the sequence $\left(f_{n}\right)$ of Fibonacci numbers as follows

$$
\widehat{f}_{n k}=\left\{\begin{array}{cll}
-\frac{f_{n+1}}{f_{n}} & , \quad k=n-1, \\
\frac{f_{n}}{f_{n+1}} & , \quad k=n, \\
0 \quad & , \quad 0 \leq k<n-1 \text { or } k>n
\end{array}\right.
$$

for all $k, n \in \mathbb{N}$. Also, in [33], Kara et al. have characterized some classes of compact operators on the spaces $\ell_{p}(\widehat{F})$ and $\ell_{\infty}(\widehat{F})$, where $1 \leq p<\infty$. Furthermore, quite recently, the sequence spaces $\lambda(\widehat{F})$ and $\mu(\widehat{F}, p)$ studied by Başarır et al. [34], and Kara and Demiriz [35], respectively, where $\lambda \in\left\{c_{0}, c\right\}$ and $\mu \in\left\{c_{0}, c, \ell_{\infty}\right\}$.

In this paper, we introduce new sequence space $\widehat{c}^{f}$ that consist of all sequences whose $\widehat{F}$-transforms in the space $\widehat{c}$. Also, we show that $\widehat{c}^{f}$ is linearly isomorphic to the space $\widehat{c}$. Further, we compute the $\beta$-dual of the space $\widehat{c}^{f}$ and characterize the classes of infinite matrices related to sequence space $\widehat{c}^{f}$. Finally, we have defined Fibonacci Core $(\widehat{F}-$ core $)$ of a sequence and characterized some class of matrices for which $\widehat{F}-\operatorname{core}(A x) \subseteq \mathcal{K}-\operatorname{core}(x)$ and $\widehat{F}-\operatorname{core}(A x) \subseteq s t_{A}-\operatorname{core}(x)$ for all $x \in \ell_{\infty}$.

\section{The Sequence Space $\widehat{c}^{f}$ Derived by the Domain of the Matrix $\widehat{F}$}

In this section, we define the sequnce space $\widehat{c}^{f}$ and give an isomorphism between the spaces $\widehat{c}^{f}$ and $\widehat{c}$ respectively. Later, we determine the $\beta$-dual of the space $\widehat{c}^{f}$.

We introduce the sequence space $\widehat{c}^{f}$ as the set of all sequence whose $\widehat{F}-$ transforms are in the space $\widehat{c}$, that is 


$$
\widehat{c}^{f}=\left\{x=x_{k} \in \omega: \exists \alpha \in \mathbb{C} \ni \lim _{m \rightarrow \infty} \sum_{j=0}^{m} \frac{y_{n+j}}{m+1}=\alpha \text { uniformly in } n\right\},
$$

where $y=\left(y_{n}\right)$ is the $\widehat{F}$-transform of a sequence $x=\left(x_{n}\right)$, i.e.,

$$
y_{n}=\widehat{F}_{n}(x)=\left\{\begin{array}{cc}
x_{0} & , \quad n=0 \\
\frac{f_{n}}{f_{n+1}} x_{n}-\frac{f_{n+1}}{f_{n}} x_{n-1} & , \quad n \geq 1
\end{array}\right.
$$

It is clear that the space $\widehat{c}^{f}$ can be redefined as

$$
\widehat{c}^{f}=\widehat{c}_{\widehat{F}} .
$$

Now, we may give following theorem concerning the isomorphism between the spaces $\widehat{c}^{f}$ and $\widehat{c}$.

Theorem 2.1. The sequence space $\widehat{c}^{f}$ is linearly isomorphic to the space $\widehat{c}$, that is, $\widehat{c}^{f} \cong \widehat{c}$.

Proof. To prove this, we should show the existence of a linear bijection between the spaces $\widehat{c}^{f}$ and $\widehat{c}$. Consider the transformation $L$ defined, with the notation of (2.1) from $\widehat{c}^{f}$ to $\widehat{c}$ by $x \longmapsto y=L x=\widehat{F} x$. The linearity of $L$ is clear. Further, it is trivial that $x=\theta$ whenever $L x=\theta$ and hence is injective.

Let us take any $y=\left(y_{k}\right)$ and consider the sequence $x=\left(x_{k}\right)$ using the inverse $\widehat{F}^{-1}$ defined by

$$
x_{k}=\widehat{F}_{k}^{-1} y=\sum_{j=0}^{k} \frac{f_{k+1}^{2}}{f_{j} f_{j+1}} y_{j}
$$

for all $k \in \mathbb{N}$. Then, we have

$$
\frac{f_{n}}{f_{n+1}} x_{n}-\frac{f_{n+1}}{f_{n}} x_{n-1}=\frac{f_{k}}{f_{k+1}} \sum_{j=0}^{k} \frac{f_{k+1}^{2}}{f_{j} f_{j+1}} y_{j}-\frac{f_{k+1}}{f_{k}} \sum_{j=0}^{k-1} \frac{f_{k}^{2}}{f_{j} f_{j+1}} y_{j}=y_{k}
$$

for all $k \in \mathbb{N}$ which leads us to the fact that

$$
\begin{aligned}
\lim _{m \rightarrow \infty} \sum_{j=0}^{m} \frac{\frac{f_{n+j}}{f_{n+1+j}} x_{n+j}-\frac{f_{n+1+j}}{f_{n+j}} x_{n-1+j}}{m+1} & =\lim _{m \rightarrow \infty} \frac{1}{m+1} \sum_{j=0}^{m} y_{k+j} \text { uniformly in } k \\
& =\hat{c}-\lim y_{k} .
\end{aligned}
$$


This means that $x=\left(x_{k}\right) \in \widehat{c}^{f}$. Consequently, we see from here that $L$ is surjective. Hence, $L$ is a linear bijection, which therefore says that the spaces $\widehat{c}^{f}$ and $\widehat{c}$ are linearly isomorphic as was desired.

If a normed sequence space $X$ contains a sequence $\left(b_{n}\right)$ with the property that for every $x \in X$ there is a unique sequence of scalars $\left(\alpha_{n}\right)$ such that

$$
\lim _{n \rightarrow \infty}\left\|x-\left(a_{0} b_{0}+a_{1} b_{1}+\ldots+a_{n} b_{n}\right)\right\|=0
$$

then $\left(b_{n}\right)$ is called a Schauder basis (or briefly basis) for $X$. The series $\sum \alpha_{k} b_{k}$ which has the sum $\mathrm{x}$ is then called the expansion of $x$ with respect to $\left(b_{n}\right)$, and written as $x=\sum \alpha_{k} b_{k}[36]$.

Lemma 2.2. ([6, Corollarry 3.3]) The Banach space $\widehat{c}$ has no Schauder basis.

Since the matrix $\widehat{F}$ is a triangle and the space $\widehat{c}$ has no Schauder basis by Lemma 2.2 , we have from [37, Remark 2.4]:

Corollary 2.3. The space $\widehat{c}^{f}$ has no Schauder basis.

Let $X$ and $Y$ be given sequence spaces. Then, the set $S(X, Y)$ defined by

$$
S(X, Y)=\left\{z=\left(z_{k}\right) \in \omega: x z=\left(x_{k} z_{k}\right) \in Y \text { for all } x=\left(x_{k}\right) \in X\right\}
$$

is called the multiplier space of the spaces $X$ and $Y$. Then, the $\beta$-dual of a sequence $X$, denoted by $X^{\alpha}$, is defined by $X^{\alpha}=S(X, c s)$.

The following lemma is essential to compute $\beta$-dual of the space $\widehat{c}^{f}$.

Lemma 2.4. ([16]) $A=\left(a_{n k}\right) \in(\widehat{c}: c)$ if and only if there are $\alpha_{k}, \alpha \in \mathbb{C}$ such that

$$
\begin{gathered}
\lim _{n \rightarrow \infty} a_{n k}=\alpha_{k} \text { for each } k \in \mathbb{N}, \\
\lim _{n \rightarrow \infty} \sum_{k} a_{n k}=\alpha, \\
\lim _{n \rightarrow \infty} \sum_{k}\left|\Delta\left(a_{n k}-\alpha_{k}\right)\right|=0, \\
\sup _{n \in \mathbb{N}} \sum_{k}\left|a_{n k}\right|<\infty,
\end{gathered}
$$

where $\Delta\left(a_{n k}-\alpha_{k}\right)=\left(a_{n k}-\alpha_{k}\right)-\left(a_{n, k+1}-\alpha_{k+1}\right)(n, k \in \mathbb{N})$. 
Theorem 2.5. Define the sets $d_{1}, d_{2}, d_{3}, d_{4}$ and $d_{5}$ by

$$
\begin{gathered}
d_{1}=\left\{a=\left(\alpha_{k}\right) \in \omega: \lim _{n \rightarrow \infty} \sum_{j=k}^{n} \frac{f_{j+1}^{2}}{f_{k} f_{k+1}} a_{j} \text { exists }\right\}, \\
d_{2}=\left\{a=\left(\alpha_{k}\right) \in \omega: \lim _{n \rightarrow \infty} \sum_{k=0}^{n}\left(\sum_{j=k}^{n} \frac{f_{j+1}^{2}}{f_{k} f_{k+1}} a_{j}\right) \text { exists }\right\}, \\
d_{3}=\left\{a=\left(\alpha_{k}\right) \in \omega: \lim _{n \rightarrow \infty} \sum_{k=0}^{n}\left|\sum_{i=k+1}^{\infty} \frac{f_{i+1}^{2}}{f_{k} f_{k+1}} a_{i}\right|=0\right\}, \\
d_{4}=\left\{a=\left(\alpha_{k}\right) \in \omega: \lim _{n \rightarrow \infty} \sum_{k=n+1}^{\infty}\left|\sum_{i=k+1}^{\infty} \frac{f_{i+1}^{2}}{f_{k} f_{k+1}} a_{i}\right|=0\right\},
\end{gathered}
$$

and

$$
d_{5}=\left\{a=\left(\alpha_{k}\right) \in \omega: \sup _{n \in \mathbb{N}} \sum_{k=0}^{n}\left|\sum_{j=k}^{n} \frac{f_{j+1}^{2}}{f_{k} f_{k+1}} a_{j}\right|<\infty\right\} .
$$

Then,

$$
\left\{\widehat{c}^{f}\right\}^{\beta}=\cap_{i=1}^{5} d_{i} .
$$

Proof. Take any $a=\left(\alpha_{k}\right) \in \omega$ and consider the equality obtained with (2.2) that

$$
\sum_{k=0}^{n} a_{k} x_{k}=\sum_{k=0}^{n} a_{k}\left(\sum_{j=0}^{n} \frac{f_{k+1}^{2}}{f_{j} f_{j+1}} y_{j}\right)=\sum_{k=0}^{n}\left(\sum_{j=k}^{n} \frac{f_{j+1}^{2}}{f_{k} f_{k+1}} a_{j}\right) y_{k}=D_{n}(y),
$$

for all $n \in \mathbb{N}$, where $D=\left(d_{n k}\right)$ is defined by

$$
d_{n k}=\left\{\begin{array}{cc}
\sum_{j=k}^{n} \frac{f_{j+1}^{2}}{f_{k} f_{k+1}} a_{j} & (0 \leq k \leq n) \\
0 & (k>n)
\end{array} ; n, k \in \mathbb{N} .\right.
$$

Then, one can easily see from (2.7) that $a x=\left(\alpha_{k} x_{k}\right) \in c s$ whenever $x=\left(x_{k}\right) \in \widehat{c}^{f}$ if and only if $D y \in c$ whenever $y=\left(y_{k}\right) \in \widehat{c}$. Therefore, we derive from Lemma 2.4 that $a x=\left(\alpha_{k} x_{k}\right) \in c s$ whenever $x=\left(x_{k}\right) \in \widehat{c}^{f}$ if and only if $a=\left(\alpha_{k}\right) \in \cap_{i=1}^{5} d_{i}$. This means that $\left\{c^{f}\right\}^{\beta}=\cap_{i=1}^{5} d_{i}$, which concludes the proof.

\section{Some Matrix Transformations Related to the Sequence Space $\widehat{c}^{f}$}

In the present section, we characterize the matrix transformations from $\widehat{c}^{f}$ into any given sequence space $X$ and from a given sequence space $X$ into $\widehat{c}^{f}$.

Throughout this section we shall write for brevity that 


$$
\begin{gathered}
\tilde{a}_{n k}=\sum_{j=k}^{\infty} \frac{f_{j+1}^{2}}{f_{k} f_{k+1}} a_{n j}, \\
\bar{a}_{n k}=\frac{f_{n}}{f_{n+1}} a_{n k}-\frac{f_{n+1}}{f_{n}} a_{n-1, k}, \\
a(n, k)=\sum_{j=0}^{n} a_{j k}
\end{gathered}
$$

and

$$
a(n, k, m)=\frac{1}{m+1} \sum_{j=0}^{m} a_{n+j, k} \quad(m \in \mathbb{N})
$$

for all $k, n \in \mathbb{N}$. Also, since $\widehat{c}^{f} \cong \widehat{c}$, it is trivial that the equivalance " $x \in \widehat{c}^{f}$ if and only if $y \in \widehat{c}$ ' holds.

Now, we give the following two theroems to determine matrix classes on the space $\widehat{c}^{f}$. To prove these theorems, we follow the similar way due to Başar and Kiriş̧̧i [6].

Theorem 3.1. Suppose that the entries of the infinite matrices $A=\left(a_{n k}\right)$ and $T=\left(t_{n k}\right)$ are connected with the relation

$$
t_{n k}=\tilde{a}_{n k}
$$

for all $k, n \in \mathbb{N}$ and $X$ be any given sequence space. Then, $A \in\left(\widehat{c}^{f}: X\right)$ if and only if $\left\{a_{n k}\right\}_{k \in \mathbb{N}} \in\left\{\widehat{c}^{f}\right\}^{\beta}$ for all $n \in \mathbb{N}$ and $T \in(\widehat{c}: X)$.

Proof. Let $X$ be any given sequence space. Assume that (3.1) holds for the matrices $A=\left(a_{n k}\right)$ and $T=\left(t_{n k}\right)$, and take into account that the spaces $\widehat{c}^{f}$ and $\widehat{c}$ are linearly isomorphic.

Now, let $A \in\left(\widehat{c}^{f}: X\right)$ and take $y=\left(y_{k}\right) \in \widehat{c}$. Then, $T \widehat{F}$ exist and $\left\{a_{n k}\right\}_{k \in \mathbb{N}} \in$ $\cap_{i=1}^{5} d_{i}$ which yields $\left\{t_{n k}\right\}_{k \in \mathbb{N}} \in \ell_{1}$ for each $n \in \mathbb{N}$. Hence, $T y$ exist and thus

$$
\sum_{k} t_{n k} y_{k}=\sum_{k} a_{n k} x_{k} \text { for all } n \in \mathbb{N}
$$

and we obtain from (3.1) that $T y=A x$, which leads us to consequence $T \in(\widehat{c}: Y)$.

Conversely, let $\left\{a_{n k}\right\}_{k \in \mathbb{N}} \in\left\{\widehat{c}^{f}\right\}^{\beta}$ for all $n \in \mathbb{N}$ and $T \in(\widehat{c}: X)$ hold, and take $x=\left(x_{k}\right) \in \widehat{c}^{f}$. Then, $A x$ exists. Therefore, we obtain from the equality

$$
\sum_{k=0}^{m} a_{n k} x_{k}=\sum_{k=0}^{m}\left[\sum_{j=k}^{m} \frac{f_{j+1}^{2}}{f_{k} f_{k+1}} a_{n j}\right] y_{k} ; \quad \text { for all } n \in \mathbb{N}
$$

as $m \rightarrow \infty$ that $T y=A x$ and this shows that $A \in\left(\widehat{c}^{f}: X\right)$. 
Theorem 3.2. Suppose that the entries of the infinite matrices $A=\left(a_{n k}\right)$ and $R=\left(r_{n k}\right)$ are connected with the relation $r_{n k}=\bar{a}_{n k}$ for all $k, n \in \mathbb{N}$ and $X$ be given sequence space. Then, $A \in\left(X: \widehat{c}^{f}\right)$ if and only if $R \in(X: \widehat{c})$.

Proof. Let $x=\left(x_{k}\right) \in X$ and consider the following equality

$$
\begin{aligned}
\{\widehat{F}(A x)\}_{n} & =\frac{f_{n}}{f_{n+1}}(A x)_{n}-\frac{f_{n+1}}{f_{n}}(A x)_{n-1} \\
& =\frac{f_{n}}{f_{n+1}} \sum_{k} a_{n k} x_{k}-\frac{f_{n+1}}{f_{n}} \sum_{k} a_{n-1, k} x_{k} \\
& =\sum_{k}\left(\frac{f_{n}}{f_{n+1}} a_{n k}-\frac{f_{n+1}}{f_{n}} a_{n-1, k}\right) x_{k}=(R x)_{n}
\end{aligned}
$$

for all $n \in \mathbb{N}$, which yields by to the generalized limit that $A x \in \widehat{c}^{f}$ if and only if $R x \in \widehat{c}$. This completes the proof.

Now, we give the following conditions:

$$
\begin{aligned}
& \sup _{n \in \mathbb{N}} \sum_{k}\left|\Delta a_{n k}\right|<\infty \\
& \lim _{k \rightarrow \infty} a_{n k}=0 \text { for each fixed } n \in \mathbb{N} \text {, } \\
& \widehat{c}-\lim a_{n k}=\alpha_{k} \text { exists for each fixed } k \in \mathbb{N} \text {, } \\
& \lim _{m \rightarrow \infty} \sum_{k}\left|a(n, k, m)-\alpha_{k}\right|=0 \text { uniformly in } n, \\
& \widehat{c}-\lim \sum_{k} a_{n k}=\alpha, \\
& \lim _{m \rightarrow \infty} \sum_{k}\left|\Delta\left[a(n, k, m)-\alpha_{k}\right]\right|=0 \text { uniformly in } n, \\
& \lim _{q \rightarrow \infty} \sum_{k} \frac{1}{q+1}\left|\sum_{i=0}^{q} \Delta\left[a(n+i, k)-\alpha_{k}\right]\right|=0 \text { uniformly in } n,
\end{aligned}
$$




$$
\begin{gathered}
\sup _{n \in \mathbb{N}} \sum_{k}|a(n, k)|<\infty, \\
\sum_{n} a_{n k}=\alpha_{k} \text { for each fixed } n \in \mathbb{N}, \\
\sum_{n} \sum_{k} a_{n k}=\alpha, \\
\lim _{n \rightarrow \infty} \sum_{k}\left|\Delta\left[a(n, k)-\alpha_{k}\right]\right|=0 .
\end{gathered}
$$

Prior to giving some results as an application of this idea, we give the following basic lemma, which is the collection of the characterizations of matrix transformations related to almost convergence.

Lemma 3.3.([6]) Let $A=\left(a_{n k}\right)$ be an infinite matrix. Then, the following statements hold:

(i) $A=\left(a_{n k}\right) \in\left(\widehat{c}: \ell_{\infty}\right)$ if and only if (2.6) holds.

(ii) $A=\left(a_{n k}\right) \in\left(\ell_{\infty}: \widehat{c}\right)$ if and only if (2.6), (3.4) and (3.5) hold.

(iii) $A=\left(a_{n k}\right) \in(\widehat{c}: \hat{c})$ if and only if (2.6), (3.4), (3.6) and (3.7) hold.

(iv) $A=\left(a_{n k}\right) \in(c: \widehat{c})$ if and only if (2.6), (3.4) and (3.6) hold.

(v) $A=\left(a_{n k}\right) \in(b s: \widehat{c})$ if and only if (3.2), (3.3), (3.4) and (3.8) hold.

(vi) $A=\left(a_{n k}\right) \in(c s: \widehat{c})$ if and only if (3.2) and (3.4) hold.

(vii) $A=\left(a_{n k}\right) \in(\widehat{c}: c s)$ if and only if (3.9) - (3.12) hold.

Then, by using Theorems 3.1 and 3.2 with Lemmas 2.4 and 3.3, we have the following corollaries.

Corollary 3.4. The following statements hold:

(i) $A=\left(a_{n k}\right) \in\left(\widehat{c}^{f}: \ell_{\infty}\right)$ if and only if $\left\{a_{n k}\right\}_{k \in \mathbb{N}} \in\left\{\widehat{c}^{f}\right\}^{\beta}$ for all $n \in \mathbb{N}$ and (2.6) hold with $\tilde{a}_{n k}$ instead of $a_{n k}$.

(ii) $A=\left(a_{n k}\right) \in\left(\widehat{c}^{f}: c\right)$ if and only if $\left\{a_{n k}\right\}_{k \in \mathbb{N}} \in\left\{\widehat{c}^{f}\right\}^{\beta}$ for all $n \in \mathbb{N}$ and (2.3) - (2.6) hold with $\tilde{a}_{n k}$ instead of $a_{n k}$.

(iii) $A=\left(a_{n k}\right) \in\left(\widehat{c}^{f}: \widehat{c}\right)$ if and only if $\left\{a_{n k}\right\}_{k \in \mathbb{N}} \in\left\{\widehat{c}^{f}\right\}^{\beta}$ for all $n \in \mathbb{N}$ and (2.6), (3.4), (3.6) and (3.7) hold with $\tilde{a}_{n k}$ instead of $a_{n k}$.

(iv) $A=\left(a_{n k}\right) \in\left(\widehat{c}^{f}:\right.$ bs) if and only if $\left\{a_{n k}\right\}_{k \in \mathbb{N}} \in\left\{\widehat{c}^{f}\right\}^{\beta}$ for all $n \in \mathbb{N}$ and 
(3.9) holds.

(v) $A=\left(a_{n k}\right) \in\left(\widehat{c}^{f}: c s\right)$ if and only if $\left\{a_{n k}\right\}_{k \in \mathbb{N}} \in\left\{\widehat{c}^{f}\right\}^{\beta}$ for all $n \in \mathbb{N}$ and (3.9) - (3.12) hold with $\tilde{a}_{n k}$ instead of $a_{n k}$.

Corollary 3.5. The following statements hold:

(i) $A=\left(a_{n k}\right) \in\left(\ell_{\infty}: \widehat{c}^{f}\right)$ if and only if (2.6), (3.4) and (3.5) hold with $\bar{a}_{n k}$ instead of $a_{n k}$.

(ii) $A=\left(a_{n k}\right) \in\left(c: \widehat{c}^{f}\right)$ if and only if (2.6), (3.4) and (3.6) hold with $\bar{a}_{n k}$ instead of $a_{n k}$.

(iii) $A=\left(a_{n k}\right) \in\left(\widehat{c}: \widehat{c}^{f}\right)$ if and only if (2.6), (3.4), (3.6) and (3.7) hold with $\bar{a}_{n k}$ instead of $a_{n k}$.

(iv) $A=\left(a_{n k}\right) \in\left(b s: \widehat{c}^{f}\right)$ if and only if (3.2), (3.3), (3.4) and (3.8) hold with $\bar{a}_{n k}$ instead of $a_{n k}$.

(v) $A=\left(a_{n k}\right) \in\left(c s: \widehat{c}^{f}\right)$ if and only if (3.2) and (3.4) hold with $\bar{a}_{n k}$ instead of $a_{n k}$.

\section{Fibonacci Core}

Using the convergence domain of the matrix $\widehat{F}=\left(f_{n k}\right)$, the new sequence spaces $c_{0}(\widehat{F})$ and $c(\widehat{F})$ have been constructed and their some properties have been investigated in [34]. In this section we will consider the sequences with complex entries and by $\ell_{\infty}(\mathbb{C})$ denote the space of all bounded complex valued sequences.

Following Knopp, a core theorem is characterized a class of matrices for which the core of the transformed sequence is included by the core of the original sequence. For example Knopp Core Theorem [22, p.138] states that $\mathcal{K}-\operatorname{core}(A x) \subseteq \mathcal{K}-$ $\operatorname{core}(x)$ for all real valued sequences $x$ whenever $A$ is a positive matrix in the class $(c: c)_{\text {reg }}$.

Here, we will define Fibonacci core (or $\widehat{F}$-core) of a complex valued sequence and characterize the class of matrices to yield $\widehat{F}-\operatorname{core}(A x) \subseteq \mathcal{K}-\operatorname{core}(x)$ and $\widehat{F}-\operatorname{core}(A x) \subseteq s t-\operatorname{core}(x)$ for all $x \in \ell_{\infty}(\mathbb{C})$.

Now, let us write

$$
y_{n}(x)=\widehat{F}_{n}(x)= \begin{cases}\frac{f_{0}}{f_{1}} x_{0}=x_{0}, & (n=0), \\ \frac{f_{n}}{f_{n+1}} x_{n}-\frac{f_{n+1}}{f_{n}} x_{n-1}, & (n \geq 1),\end{cases}
$$

where $n \in \mathbb{N}$. Then, we can define $\widehat{F}-$ core of a complex sequence as follows: 
Definition 4.1. Let $H_{n}$ be the least closed convex hull containing $y_{n}(x), y_{n+1}(x)$, $y_{n+2}(x), \ldots$ Then, $\widehat{F}-$ core of $x$ is the intersection of all $H_{n}$, i.e.,

$$
\widehat{F}-\operatorname{core}(x)=\bigcap_{n=1}^{\infty} H_{n} .
$$

Note that, actually, we define $\widehat{F}$ - core of $x$ by the $\mathcal{K}$ - core of the sequence $\left(y_{n}(x)\right)$. Hence, we can construct the following theorem which is an analogue of $\mathcal{K}-$ core, [23]:

Theorem 4.2. For any $z \in \mathbb{C}$, let

$$
G_{x}(z)=\left\{\omega \in \mathbb{C}:|\omega-z| \leq \limsup _{n}\left|y_{n}(x)-z\right|\right\} .
$$

Then, for any $x \in \ell_{\infty}$,

$$
\widehat{F}-\operatorname{core}(x)=\bigcap_{z \in \mathbb{C}} G_{x}(z)
$$

Now, we prove some lemmas which will be useful to the main results of this section. To do these, we need to characterize the classes $(c: c(\widehat{F}))_{\text {reg }}$ and $(\operatorname{st}(A) \cap$ $\left.\ell_{\infty}: c(\widehat{F})\right)_{r e g}$. For brevity, in what follows we write $\widetilde{b}_{n k}$ in place of

$$
\frac{f_{n}}{f_{n+1}} b_{n k}-\frac{f_{n+1}}{f_{n}} b_{n-1, k} ; \quad(n \geq 1) .
$$

Lemma 4.3. $B \in\left(\ell_{\infty}: c(\widehat{F})\right)$ if and only if

$$
\begin{gathered}
\|B\|=\sup _{n} \sum_{k}\left|\tilde{b}_{n k}\right|<\infty, \\
\lim _{n} \tilde{b}_{n k}=\alpha_{k} \text { for each } k, \\
\lim _{n} \sum_{k}\left|\tilde{b}_{n k}-\alpha_{k}\right|=0 .
\end{gathered}
$$

Lemma 4.4. $B \in(c: c(\widehat{F}))_{\text {reg }}$ if and only if (4.1) and (4.2) of the Lemma 4.3 hold with $\alpha_{k}=0$ for all $k \in \mathbb{N}$ and

$$
\lim _{n} \sum_{k} \tilde{b}_{n k}=1 .
$$


Lemma 4.5. $B \in\left(\operatorname{st}(A) \cap \ell_{\infty}: c(\widehat{F})\right)_{\text {reg }}$ if and only if $B \in(c: c(\widehat{F}))_{\text {reg }}$ and

$$
\lim _{n} \sum_{k \in E}\left|\tilde{b}_{n k}\right|=0
$$

for every $E \subset \mathbb{N}$ with $\delta_{A}(E)=0$.

Proof. Because of $c \subset$ st $\cap \ell_{\infty}, B \in(c: c(\widehat{F}))_{\text {reg }}$. Now, for any $x \in \ell_{\infty}$ and a set $E \subset \mathbb{N}$ with $\delta(E)=0$, let us define the sequence $z=\left(z_{k}\right)$ by

$$
z_{k}=\left\{\begin{array}{cc}
x_{k}, & k \in E \\
0, & k \notin E .
\end{array}\right.
$$

Then, since $z \in s t_{0}, A z \in c_{0}(\widehat{F})$, where $c_{0}(\widehat{F})$ is the space of sequences which the $\widehat{F}$ - transforms of them in $c_{0}$. Also, since

$$
\sum_{k} \tilde{b}_{n k} z_{k}=\sum_{k \in E} \tilde{b}_{n k} x_{k}
$$

the matrix $D=\left(d_{n k}\right)$ defined by $d_{n k}=\tilde{b}_{n k} \quad(k \in E)$ and $d_{n k}=0 \quad(k \notin E)$ is in the class $\left(\ell_{\infty}: c(\widehat{F})\right)$. Hence, the necessity of (4.5) follows from Lemma 4.3.

Conversely, let $x \in \operatorname{st}(A) \cap \ell_{\infty}$ with $s t_{A}-\lim x=l$. Then, the set $E$ defined by $E=\left\{k:\left|x_{k}-l\right| \geq \varepsilon\right\}$ has density zero and $\left|x_{k}-l\right| \leq \varepsilon$ if $k \notin E$. Now, we can write

$$
\sum_{k} \tilde{b}_{n k} x_{k}=\sum_{k} \tilde{b}_{n k}\left(x_{k}-l\right)+l \sum_{k} \tilde{b}_{n k}
$$

Since

$$
\left|\sum_{k} \tilde{b}_{n k}\left(x_{k}-l\right)\right| \leq\|x\| \sum_{k \in E}\left|\tilde{b}_{n k}\right|+\varepsilon \cdot\|B\|,
$$

letting $n \rightarrow \infty$ in (4.6) and using (4.4) with (4.5), we have

$$
\lim _{n} \sum_{k} \tilde{b}_{n k} x_{k}=l
$$

This implies that $B \in\left(s t(A) \cap \ell_{\infty}: c(\widehat{F})\right)_{\text {reg }}$ and the proof is completed.

Theorem 4.6. For any $z \in \mathbb{C}$, let

$$
G_{x}(z)=\left\{\omega \in \mathbb{C}:|\omega-z| \leq \underset{n}{\limsup }\left|y_{n}(x)-z\right|\right\} .
$$

Then, for any $x \in \ell_{\infty}$,

$$
\widehat{F}-\operatorname{core}(x)=\bigcap_{z \in \mathbb{C}} G_{x}(z) .
$$


Now, we may give some inclusion theorems. Firstly, we need a lemma.

Lemma 4.7.([38, Corollary 12]) Let $A=\left(a_{n k}\right)$ be a matrix satisfying $\sum_{k}\left|a_{n k}\right|<\infty$ and $\lim _{n} a_{n k}=0$. Then, there exists an $y \in \ell_{\infty}$ with $\|y\| \leq 1$ such that

$$
\limsup _{n} \sum_{k} a_{n k} y_{k}=\limsup _{n} \sum_{k}\left|a_{n k}\right| \text {. }
$$

Theorem 4.8. Let $B \in(c: c(\widehat{F}))_{\text {reg. }}$ Then, $\widehat{F}-\operatorname{core}(B x) \subseteq \mathcal{K}-\operatorname{core}(x)$ for all $x \in \ell_{\infty}$ if and only if

$$
\lim _{n} \sum_{k}\left|\tilde{b}_{n k}\right|=1
$$

Proof. Since $B \in(c: c(\widehat{F}))_{\text {reg }}$, the matrix $\tilde{B}=\left(\tilde{b}_{n k}\right)$ is satisfy the conditions of Lemma 4.7. So, there exists a $y \in \ell_{\infty}$ with $\|y\| \leq 1$ such that

$$
\left\{\omega \in \mathbb{C}:|\omega| \leq \limsup _{n} \sum_{k} \tilde{b}_{n k} y_{k}\right\}=\left\{\omega \in \mathbb{C}:|\omega| \leq \limsup _{n} \sum_{k}\left|\tilde{b}_{n k}\right|\right\}
$$

On the other hand, since $\mathcal{K}-\operatorname{core}(y) \subseteq B_{1}(0)$, by the hypothesis

$$
\left\{\omega \in \mathbb{C}:|\omega| \leq \limsup _{n} \sum_{k}\left|\tilde{b}_{n k}\right|\right\} \subseteq B_{1}(0)=\{\omega \in \mathbb{C}:|\omega| \leq 1\}
$$

which implies (4.7).

Conversely, let $\omega \in \widehat{F}-\operatorname{core}(B x)$. Then, for any given $z \in \mathbb{C}$, we can write

$$
\begin{aligned}
& |\omega-z| \leq \lim \sup \left|y_{n}(B x)-z\right| \\
& =\limsup _{n}\left|z-\sum_{k} \tilde{b}_{n k} x_{k}\right| \\
& \leq \limsup _{n}\left|\sum_{k} \tilde{b}_{n k}\left(z-x_{k}\right)\right|+\limsup _{n}|z|\left|1-\sum_{k} \tilde{b}_{n k}\right| \\
& =\limsup _{n}\left|\sum_{k} \tilde{b}_{n k}\left(z-x_{k}\right)\right| \text {. }
\end{aligned}
$$

Now, let $\lim \sup _{k}\left|x_{k}-z\right|=l$. Then, for any $\varepsilon>0,\left|x_{k}-z\right| \leq l+\varepsilon$ whenever $k \geq k_{0}$. Hence, one can write that 


$$
\begin{aligned}
\left|\sum_{k} \tilde{b}_{n k}\left(z-x_{k}\right)\right| & =\left|\sum_{k<k_{0}} \tilde{b}_{n k}\left(z-x_{k}\right)+\sum_{k \geq k_{0}} \tilde{b}_{n k}\left(z-x_{k}\right)\right| \\
& \leq \sup _{k}\left|z-x_{k}\right| \sum_{k<k_{0}}\left|\tilde{b}_{n k}\right|+(l+\varepsilon) \sum_{k \geq k_{0}}\left|\tilde{b}_{n k}\right| \\
& \leq \sup _{k}\left|z-x_{k}\right| \sum_{k<k_{0}}\left|\tilde{b}_{n k}\right|+(l+\varepsilon) \sum_{k}\left|\tilde{b}_{n k}\right| .
\end{aligned}
$$

Therefore, applying $\lim \sup _{n}$ under the light of the hypothesis and combining (4.8) with (4.9), we have

$$
|\omega-z| \leq \limsup _{n}\left|\sum_{k} \tilde{b}_{n k}\left(z-x_{k}\right)\right| \leq l+\varepsilon
$$

which means that $\omega \in \mathcal{K}-\operatorname{core}(x)$. This completes the proof.

Theorem 4.9. Let $B \in\left(s t(A) \cap \ell_{\infty}: c(\widehat{F})\right)_{\text {reg. }}$. Then, $\widehat{F}-\operatorname{core}(B x) \subseteq s t_{A}-\operatorname{core}(x)$ for all $x \in \ell_{\infty}$ if and only if (4.7) holds.

Proof. Since $s t_{A}-\operatorname{core}(x) \subseteq \mathcal{K}-\operatorname{core}(x)$ for any sequence $x$ [39], the necessity of the condition (4.7) follows from Theorem 4.8.

Conversely, take $\omega \in \widehat{F}-\operatorname{core}(B x)$. Then, we can write again (4.8). Now; if $s t_{A}-\limsup \left|x_{k}-z\right|=s$, then for any $\varepsilon>0$, the set $E$ defined by $E=\{k$ : $\left.\left|x_{k}-z\right|>s+\varepsilon\right\}$ has density zero, (see [39]). Now, we can write

$$
\begin{aligned}
\left|\sum_{k} \tilde{b}_{n k}\left(z-x_{k}\right)\right| & =\left|\sum_{k \in E} \tilde{b}_{n k}\left(z-x_{k}\right)+\sum_{k \notin E} \tilde{b}_{n k}\left(z-x_{k}\right)\right| \\
& \leq \sup _{k}\left|z-x_{k}\right| \sum_{k \in E}\left|\tilde{b}_{n k}\right|+(s+\varepsilon) \sum_{k \notin E}\left|\tilde{b}_{n k}\right| \\
& \leq \sup _{k}\left|z-x_{k}\right| \sum_{k \in E}\left|\tilde{b}_{n k}\right|+(s+\varepsilon) \sum_{k}\left|\tilde{b}_{n k}\right| .
\end{aligned}
$$

Thus, applying the operator limsup $\sup _{n}$ and using the condition (4.7) with (4.5), we get that

$$
\limsup _{n}\left|\sum_{k} \tilde{b}_{n k}\left(z-x_{k}\right)\right| \leq s+\varepsilon .
$$

Finally, combining (4.8) with (4.10), we have

$$
|\omega-z| \leq s t_{A}-\limsup _{k}\left|x_{k}-z\right|
$$


which means that $\omega \in s t_{A}-\operatorname{core}(x)$ and the proof is completed.

As a consequence of Theorem 4.9 , we have

Corollary 4.10. Let $B \in\left(\text { st } \cap \ell_{\infty}: c(\widehat{F})\right)_{\text {reg }}$. Then, $\widehat{F}-\operatorname{core}(B x) \subseteq s t-\operatorname{core}(x)$ for all $x \in \ell_{\infty}$ if and only if (4.7) holds.

\section{References}

[1] S. Banach, Théorie des opérations linéaires. Chelsea Publishing company., New York (1978).

[2] G. G. Lorentz, A contribution to the theory of divergent sequences, Acta Math., 80(1948), 167-190.

[3] J. Boos, Classical and Modern Methods in Summability, Oxford University Press Inc, New York, 2000.

[4] M. Şengönül and K. Kayaduman, On the Riesz almost convergent sequence space, Abstr. Appl. Anal. Volume 2012, Article ID 691694, 18 pages, doi:10.1155/2012/691694.

[5] K. Kayaduman and M. Şengönül, The spaces of Cesàro almost convergent sequences and core Theorems, Acta Math. Sci., 32B(6)(2012), 2265-2278.

[6] F. Başar and M. Kirişçi, Almost convergence and generalized difference matrix, Comput. Math. Appl., 61(2011), 602-611.

[7] A. Sönmez, Almost convergence and triple band matrix, Math. Comput. Model., 57(910)(2012), 2393-2402.

[8] E. E. Kara and K. Elmaağaç, On the u-difference almost sequence space and matrix transformations, Inter. J. Modern Math. Sci., 8(1)(2013), 57-76.

[9] Mursaleen, Invariant means and some matrix transformations, Indian J. Pure Appl. Math., 25(3)(1994), 353-359.

[10] M. Mursaleen, E. Savaş, M. Aiyub and S. A. Mohuiddine, Matrix transformations between the spaces of Cesàro sequences and invariant means, Int. J. Math. Math. Sci. Volume 2006 (2006), Article ID 74319, 8 pages, doi:10.1155/IJMMS/2006/74319.

[11] J.P. Duran, Infinite matrices and almost convergence, Math. Z., 128(1972), 75-83.

[12] F. Başar and İ. Solak, Almost-coercive matrix transformations, Rend. Mat. Appl., (7)11(2)(1991), 249-256.

[13] F. Başar and R. Çolak, Almost-conservative matrix transformations, Turkish J. Math., 13(3)(1989), 91-100.

[14] F. Başar, f-conservative matrix sequences, Tamkang J. Math., 22(2)(1991), 205212.

[15] J. P. King, Almost summable sequences, Proc. Amer. Math. Soc., 17(1966), 12191225.

[16] J. A. Siddıqi, Infinite matrices summing every almost periodic sequences, Pacific. J. Math., 39(1)(1971), 235-251. 
[17] Qamaruddin and S. A. Mohuiddine, Almost convergence and some matrix transformations, Filomat, 21(2)(2007), 261-266.

[18] S. Nanda, Matrix transformations and almost boundedness, Glasnik Mat., 34(14)(1979), 99-107.

[19] S. A. Gupkari, Some new sequence spaces and almost convergence, Filomat, $\mathbf{2 2 ( 2 ) ( 2 0 0 8 ) , ~ 5 9 - 6 4 . ~}$

[20] Mursaleen, Invariant means and some matrix transformations, Indian J. Pure Appl. Math., 25(3)(1994), 353-359.

[21] G. Das, B. Kuttner and S. Nanda, On absolute almost convergence, J. Math. Anal. Appl., 161(1)(1991), 50-56.

[22] R. G. Cooke, Infinite Matrices and Sequence Spaces, Mcmillan, New York, 1950.

[23] A. A. Shcherbakov, Kernels of sequences of complex numbers and their regular transformations, Math. Notes, 22(1977), 948-953.

[24] H. Steinhaus, Quality control by sampling, Collog. Math., 2(1951), 98-108.

[25] J. A. Fridy and C. Orhan, Statistical core theorems, J. Math. Anal. Appl., 208(1997), $520-527$.

[26] H. S. Allen, T-transformations which leave the core of every bounded sequence invariant, J. London Math. Soc., 19(1944), 42-46.

[27] J. Connor, J. A. Fridy and C. Orhan, Core equality results for sequences, J. Math. Anal. Appl., 321(2006), 515-523.

[28] C. Çakan and H. Çoşkun, Some new inequalities related to the invariant means and uniformly bounded function sequences, Appl. Math. Lett., 20(6)(2007), 605-609.

[29] H. Çoşkun and C. Çakan, A class of statistical and $\sigma$-conservative matrices, Czechoslovak Math. J., 55(3)(2005), 791-801.

[30] H. Çoşkun, C. Çakan and M. Mursaleen, On the statistical and $\sigma$-cores, Studia Math., 154(1)(2003), 29-35.

[31] T. Koshy, Fibonacci and Lucas Numbers with Applications. Wiley, 2001.

[32] E. E. Kara, Some topological and geometrical properties of new Banach sequence spaces, J. Inequal. Appl., 2013(38)(2013), 15 pages.

[33] E. E. Kara, M. Başarır and M. Mursaleen, Compact operators on the Fibonacci difference sequence spaces $\ell_{p}(\widehat{F})$ and $\ell_{\infty}(\widehat{F})$, 1st International Eurasian Conference on Mathematical Sciences and Applications, Prishtine-Kosovo, September 3-7, 2012.

[34] M. Başarır, F. Başar and E. E. Kara, On The Spaces of Fibonacci Difference Null and Convergent Sequences, arXiv:1309.0150.

[35] E. E. Kara and S. Demiriz, Some new paranormed Fibonacci difference sequence spaces, 2st International Eurasian Conference on Mathematical Sciences and Applications, Sarajevo-Bosnia and Herzegouina, August 26-29, 2013.

[36] F. Başar, Summability Theory and Its Applications, Bentham Science Publishers, e-books, Monographs, İstanbul, 2012.

[37] A. M. Jarrah and E. Malkowsky, BK spaces, bases and linear operators, Ren. Circ. Mat. Palermo, II 52(1990), 177-191. 
[38] S. Simons, Banach limits, infinite matrices and sublinear functionals, J. Math. Anal. Appl., 26(1969), 640-655.

[39] K. Demirci, A-statistical core of a sequence, Demonstratio Math., 33(2000), 43-51. 